\title{
Comparison of pregnancy and implantation rates in zygote intrafallopian transfer and uterine embryo transfer for nontubal infertility
}

M. Agha-Hosseini, ${ }^{1}$ A. Aleyaseen, ${ }^{7}$ S. Peyvandi ${ }^{2}$ and L. Kashani ${ }^{1,3}$

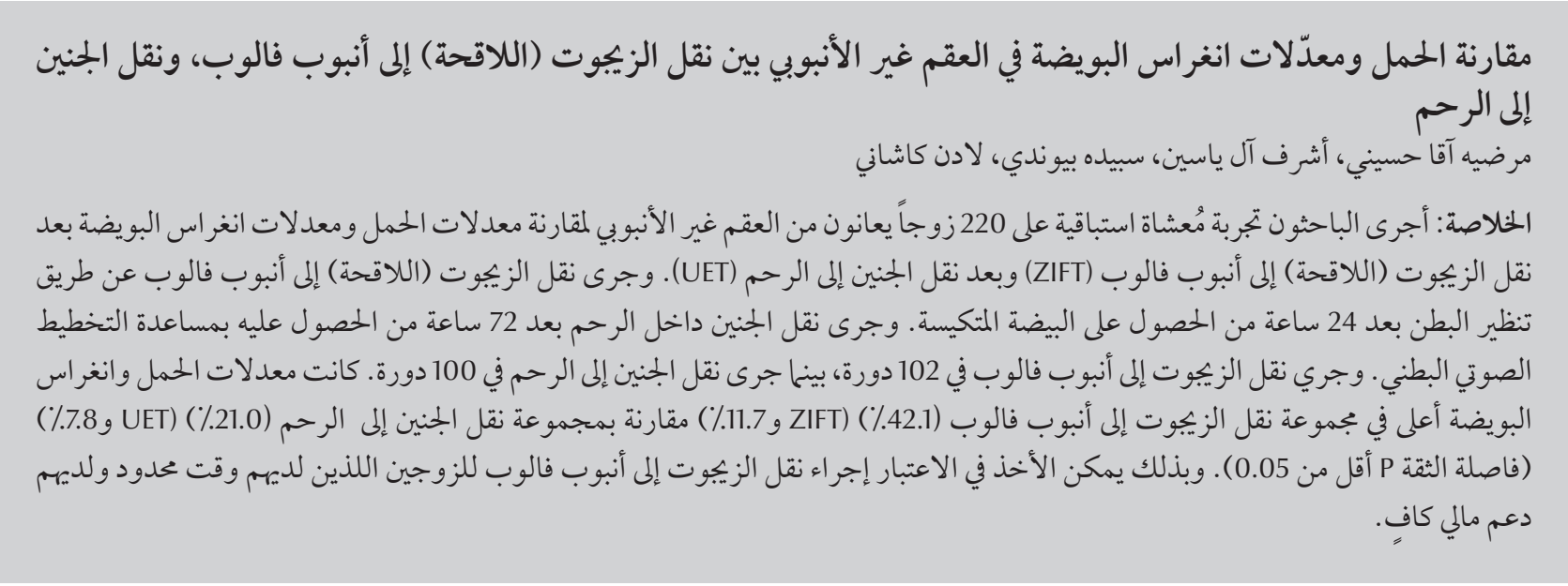

ABSTRACT We carried out a prospective randomized trial on 220 couples with nontubal factor infertility to compare pregnancy rates and implantation rates after zygote intrafallopian transfer (ZIFT) and uterine embryo transfer (UET). The zygote was transferred by laparoscopy into the fallopian tube 24 hours after oocyst retrieval. UET was performed 72 hours after retrieval with abdominal sonography guide. Transfer was performed in 102 cycles in the ZIFT and 100 cycles in the UET group. The pregnancy and implantation rates were significantly higher in the ZIFT group (42.1\% and $11.7 \%)$ than in the UET group $(21.0 \%$ and $7.8 \%)(P<0.05)$. ZIFT could be considered for couples who have limited time and adequate financial support.

Comparaison des taux de grossesse et d'implantation après un transfert intra-tubaire de zygote et un transfert intra-utérin d'embryon en cas de stérilité non tubaire

RÉSUMÉ Nous avons réalisé un essai prospectif randomisé sur 220 couples présentant une stérilité due à un facteur non tubaire afin de comparer les taux de grossesse et les taux d'implantation après un transfert intratubaire de zygote ZIFT) et un transfert intra-utérin (TIU) d'embryon. Le zygote a été transféré par cœlioscopie dans l'une des trompes de Fallope 24 heures après le prélèvement d'ovocytes, tandis que le transfert intrautérin d'embryon a été réalisé 72 heures après le prélèvement par voie vaginale avec guidage échographique. Le transfert a été effectué sur 102 cycles dans le groupe ZIFT et sur 100 cycles dans le groupe TIU. Les taux de grossesse et d'implantation étaient significativement plus élevés dans le groupe ZIFT (42,1\% et $11,7 \%$ ) que dans le groupe TIU $(21,0 \%$ et 7,8 \%) $(P<0,05)$. La méthode ZIFT pourrait être envisagée pour les couples disposant d'un temps limité et de ressources financières suffisantes.

'Infertility Centre, Dr. Shariati Hospital; ${ }^{3}$ Infertility Ward, Arash Hospital, Tehran University ofMedical Sciences, Tehran, Islamic Republic of Iran (Correspondence to L. Kashani: kashani_ladan@yahoo.co.uk).

2IVF Unit, Imam Khomeini Hospital, Mazandaran University of Medical Sciences, Sari, Islamic Republic of Iran.

Received: 10/04/07; accepted: 02/09/07 


\section{Introduction}

The ability of tubal transfer of an embryo to produce pregnancy and live birth was first demonstrated in a non-human primate model[ $[1]$.Soon afterwards thefirst successful zygote intrafallopian transfer (ZIFT) in humans was described [2]. Initial reports showed superior results using ZIFT rather than uterine embryo transfer (UET) [3-6].

Subsequent prospective clinical trials failed to support the improved efficacy in nontubal infertility $[7-10]$. Nevertheless, due to heterogeneity of study populations and differences in sample size and treatment protocols in these clinical trials, the efficacy of ZIFT over UET has not been evaluated critically.

Each technique has advantages and disadvantages. Clinical experience has shown that transcervical UET is a relatively simple and safe procedure which does not have the risks and complications associated with general anaesthesia and laparoscopy. It is a cost-effective technique and permits the selection the best cleaving embryo for transfer.

ZIFT allows confirmation of fertilization before transfer as well as exclusion of polyploid embryos. There are several additional advantages of ZIFT over UET. Early cleavage and development occur in the natural and physiological environment of the fallopian tube. The tubal environment may be potentially superior to the suboptimal conditions in artificial culture media and incubators. With ZIFT, there is better synchronization between embryonic and endometrial development. In addition, the highly favourable clinical pregnancy and live birth rates per cycle commenced and per embryo transfer, all in the range of $40 \%$, show ZIFT to be a powerful clinical tool for management of patients with repeated IVF failure [11].

Embryos have been found in the vagina after UET and some transfer procedures are more frequently associated with ectopic pregnancy $[11,12]$. However, the ZIFT procedure requires general anaesthesia and laparoscopy, increasing the cost and the risk of complications.

The aim of this study was to compare pregnancy and implantation rates after ZIFT and UET for the treatment of nontubal factor infertility.

\section{Methods}

This study was approved by Research Committee of Tehran University of Medical Sciences.

\section{Study design}

This was randomized clinical trial conducted in the infertility centre of $\mathrm{Dr}$ Shariati Hospital, Tehran University of Medical Sciences from January 2005 to February 2006.

\section{Participants}

Women with normal hysterosalpingography and/or normal laparoscopy who been candidates for embryo transfer (ET) and had easy mock ET were invited to participate in this prospective randomized trial of ZIFT versus standard UET. Exclusion criteria were: contraindications for laparoscopy; repeated implantation failure in previous cycles; azoospermia; and difficult transfer in mock ET.

Informed consent was obtained from all the couples and the advantages and disadvantages of each protocol were fully explained to the couple before entering the study. The protocol was approved by the ethics committee of Tehran University of Medical Sciences.

Sample size calculations were based on a previous report [6] that the clinical pregnancy rate in tubal transfer was $40 \%$ and in UET was 20\%. Considering these pregnancy rates, and with $\alpha=0.05$ and $\beta=20 \%$, a sample size of 90 patients in each group was calculated. As some patients do not respond to ovarian stimulation or fertilization failure may occur, we increased the sample to 110 patients in each group. The patients knew that they would be randomized to either tubal transfer or uterine transfer of embryo.

Randomization was performed after the decision to enter into the study was made and mock ET was done. A woman's age is the most important factor influencing pregnancy rates after transfer [4]. Therefore, to obtain similar demographic characteristics in the 2 groups, women were stratified by age $<35$ or 35-40 years. After this, randomization was carried out by opening a sequentially numbered sealed envelope to allocate women into the treatment protocol groups.

\section{Treatment protocol}

Ovarian stimulation was achieved with long-luteal GnRH-agonist downregulation and human menopausal gonadotrophin (HMG) or HMG + metrodine from day 3 of the menstrual cycle. For all patients oral contraception was administered on days 3-23 of the cycle preceding stimulation with doxycycline $100 \mathrm{mg}$ twice per day from day 3 for 10 days only.

The GnRh-agonist buserelin acetate (Suprefact) $500 \mu \mathrm{g}$ subcutaneous was administered from day 21 of the cycle preceding stimulation until day 3 of the menstrual cycle. The dose was then reduced to $250 \mu \mathrm{g} /$ day until the day of human chorionic gonadotropin (HCG) administration.

Follicular development was stimulated with HMG or metrodine + HMG from day 3 of the menstrual cycle. The starting dose for gonadotrophins was determined by age, body mass index and ovarian response in previous stimulation cycles. Cycle monitoring was achieved by transvaginal sonography from day 10 of the stimulation cycle and repeated every $48-72 \mathrm{~h}$ until the day of HCG administration. 
Human chronic gonadotropin $(10000 \mathrm{U})$ was given when at least 2 follicles were $>18 \mathrm{~mm}$ and other follicles were $>15 \mathrm{~mm}$. The oocytes were aspirated using an ultrasound guided transvaginal approach 34-36 hours after HCG administration. Preparation of sperm for oocyte insemination was carried out using the swim-up technique. Intracytoplasmic sperm injection was achieved for all patients. The ovarian stimulation protocol, criteria for HCG administration and laboratory handling in ZIFT and UET groups were similar.

Fertilization of recovered oocytes was assessed 14-18 hours after insemination with visualization of a 2 -pronuclear stage embryo. We performed ZIFT under general anaesthesia $24 \mathrm{~h}$ after oocyte retrieval using a 3 puncture video laparoscopic technique.

After introducing the umbilical trocor and optical equipment the abdominal cavity was surveyed. After aspirating sanguineous fluid from the pelvic cavity a maximum of 5 2-pronuclear embryos were placed $4-5 \mathrm{~cm}$ into the most accessible fallopian tube (Cook catheter).

In the UET cycles, transfer was performed $72 \mathrm{~h}$ after retrieval of the embryo at the 4-8-cell stage (grade I, grade II) using a Wallace catheter guided by transabdominal sonography to place the tip $1.5 \mathrm{~cm}$ proximal to the fundus.

The luteal phase was supported in all cycles by cyclogest suppositories $400 \mathrm{~g}$ every $8-12 \mathrm{~h}$. Serum level of $\beta$-human chorionic gonadotropin was determined 12-14 days after ET and if positive, measured again after $48 \mathrm{~h}$. If the titre had increased, the use of cyclogest was continued until 12 weeks of pregnancy.

At 5 weeks after transfer, the intrauterine gestational sac and fetal heart beat were demonstrated by vaginal sonography. The patients were followed until 12 weeks pregnancy. Clinical pregnancy was defined by the presence of gestational sac 5-6 weeks after transfer.
Ongoing pregnancy was defined as a pregnancy of $>12$ weeks gestation. The implantation rate was calculated by dividing the total number of gestation sacs by the total number of transferred embryos.

\section{Statistical analysis}

Statistical comparisons were performed with the unpaired Student $t$-test and chi-squared test.

\section{Results}

There were 110 couples in each group: with 78 women $<35$ years and 32 women $35-40$ years in each. There was no significant difference between the 2 groups regarding baseline data including duration of infertility, etiology, body mass index or number of previous assisted reproduction technology cycles (Table 1).

\begin{tabular}{|c|c|c|c|c|c|}
\hline \multirow[t]{2}{*}{ Characteristic } & \multicolumn{2}{|c|}{ ZIFT } & \multicolumn{2}{|c|}{ UET } & \multirow[t]{2}{*}{$P$} \\
\hline & No. & $\%$ & No. & $\%$ & \\
\hline Number of patients & 110 & & 110 & & \\
\hline \multicolumn{6}{|l|}{ History of: } \\
\hline Previous ART cycle & 47 & & 53 & & \\
\hline IVF or ICSI + ET & 21 & 44.7 & 31 & 58.5 & NS \\
\hline ZIFT & 26 & 55.3 & 22 & 41.5 & \\
\hline \multicolumn{6}{|l|}{ Type of infertility } \\
\hline Primary & 94 & 85.3 & 92 & 83.6 & NS \\
\hline Secondary & 16 & 14.7 & 18 & 16.4 & \\
\hline \multicolumn{6}{|l|}{ Infertility diagnosis } \\
\hline Anovulation & 14 & 12.7 & 24 & 21.8 & NS \\
\hline Male factor & 53 & 48.2 & 38 & 34.5 & NS \\
\hline Unexplained & 8 & 7.3 & 7 & 6.4 & NS \\
\hline Endometriosis & 4 & 3.6 & 5 & 4.5 & NS \\
\hline \multirow[t]{2}{*}{ Male factor + anovulation } & 31 & 28.2 & 36 & 32.7 & NS \\
\hline & Mean & SD & Mean & SD & \\
\hline Age (years) & 30.9 & 5.3 & 30.4 & 4.8 & NS \\
\hline $\mathrm{BMI}\left(\mathrm{kg} / \mathrm{m}^{2}\right)$ & 22.9 & 2.3 & 22.8 & 2.5 & NS \\
\hline Duration of infertility (years) & 8.3 & 4.4 & 8.6 & 4.7 & NS \\
\hline No. of previous ART cycles & 1.29 & 0.54 & 1.30 & 0.46 & NS \\
\hline
\end{tabular}

$A R T=$ assisted reproduction technology; IVF = in vitro fertilization; ICSI = intracytoplasmic sperm injection;

$B M I=$ body mass index $; S=$ standard deviation .

$N S=$ not significant $(P>0.05)$.
Clinical data and treatment cycle characteristic were similar in the ZIFT and UET groups and are presented in Tables 1 and 2. In the ZIFT group, 6 patients did not respond to ovarian stimulation and in 2 patients fertilization failure occurred. In the UET group 5 patients were poor responders and in 5 patients fertilization failure occurred. So analysis was performed in 102 cycles in the ZIFT and 100 cycles in the UET.

There was no significant difference between the 2 groups regarding treatment protocol, stimulation duration, number of HMG ampoules, number of oocytes retrieved and number of embryos transferred. Serum estradiol level and endometrial thickness on the day of HCG injection was similar in the 2 groups.

Clinical pregnancy rate per transfer, $42.1 \%$ (43/102); ongoing pregnancy rate per transfer, $35.3 \%$ (36/102); and 


\begin{tabular}{|c|c|c|c|c|c|}
\hline \multicolumn{6}{|c|}{$\begin{array}{l}\text { Table } 2 \text { Stimulation cycles characteristic in the zygote intrafallopian transfer (ZIFT) } \\
\text { and uterine embryo transfer (UET) groups }\end{array}$} \\
\hline Characteristic & \multicolumn{2}{|c|}{ ZIFT } & \multicolumn{2}{|c|}{ UET } & $P$ \\
\hline No. of patients & \multicolumn{2}{|c|}{110} & \multicolumn{2}{|c|}{110} & \\
\hline No. of cycles with transfer & \multicolumn{2}{|c|}{102} & \multicolumn{2}{|c|}{100} & \\
\hline \multirow[t]{2}{*}{ No. (\%) of cycles cancelled } & \multicolumn{2}{|c|}{$8(7.2)$} & \multicolumn{2}{|c|}{$10(9.0)$} & NS \\
\hline & Mean & SD & Mean & SD & \\
\hline No. gonadotropine ampoules & 16.38 & 7.13 & 36.88 & 12.06 & NS \\
\hline Stimulation duration (d) & 10.7 & 1.79 & 10.8 & 1.78 & NS \\
\hline Serum $\mathrm{E}_{2}$ on HCG day $(\mathrm{pg} / \mathrm{ml})$ & 2279.1 & 1199.0 & 2296.5 & 7209.0 & NS \\
\hline $\begin{array}{l}\text { Endometrial thickness on HCG } \\
\text { day }(\mathrm{mm})\end{array}$ & 10.59 & 2.07 & 10.48 & 2.25 & NS \\
\hline No. oocytes retrieved & 7.22 & 3.00 & 6.65 & 3.17 & NS \\
\hline No. oocytes fertilized & 4.07 & 2.30 & 3.82 & 2.14 & NS \\
\hline No. embryos transferred & 4.06 & 2.02 & 3.87 & 1.61 & NS \\
\hline
\end{tabular}

$E_{2}=$ estradiol; $H C G=$ human chorionic gonadotrophin; $S D=$ standard deviation . $N S=$ not significant $(P>0.05)$.

implantation rate, $11.7 \%(57 / 488)$ were significantly higher in the ZIFT group than the UET group $[21 \%(21 / 100)$, $20 \%(20 / 100)$ and $7.8 \%(27 / 343)$ respectively] $(P<0.05)$. Abortion occurred in 7 (6.9\%) women in the ZIFT group and 1 (1\%) woman in the UET group. In those in whom abortion occurred, 2 had triplet pregnancy and 5 had twin pregnancy.

Ectopic pregnancy occurred in 1 patient in the ZIFT group; no ectopic pregnancy occurred in the UET group. The data regarding pregnancies that went to term are presented in Table 3. No triplet pregnancies went to term in the either group. There was no significant difference between the 2 groups in terms of multiple pregnancies (Table 3 ).

\section{Discussion}

This prospective randomized study demonstrated higher rates for clinical pregnancy, ongoing pregnancy and implantation with ZIFT than with UET. These findings agree with those of Pool et al. [6]. In our study, clinical pregnancy rate per transfer was $42.1 \%$ in ZIFT and $21 \%$ in UET. Ongoing pregnancy rate per transfer was $35.3 \%$ in ZIFT versus $20 \%$ in UET. In the first prospective study of this issue, reported pregnancy rate per transfer was $47.7 \%$ for in vitro fertilization and $37.9 \%$ for ZIFT [7]. The difference between their results and ours may be related to differences in sample size and treatment protocol. [7].

A Canadian study failed to demonstrate any obvious benefit of tubal transfer compared to uterine transfer [8]. Clinical pregnancy per retrieval was $12 \%$ with ZIFT and $26.5 \%$ with UET [8] although the treatment protocol was not comparable for all patients. Ovarian stimulation was achieved with clomiphen + HMG, HMG or GnRHagonist in short protocol. Meta-analysis of prospective clinical trials did not demonstrate any differences in clinical pregnancy rate per transfer and implantation rate between ZIFT and UET (36.5\%, 15\% in ZIFT versus 31.4\%, 12\% in UET), although there was a tendency towards a higher pregnancy rate with ZIFT [10].

In our study, there were no differences between the 2 groups regarding demographic and baseline data.

Our findings suggest that the difference in clinical pregnancy between the 2 groups is due to differences in the transfer techniques. One of the factors that most affected UET cycles was quality of transfer. Clinical pregnancy and implantation rates were significantly higher with ZIFT than with UET.

It has been reported that UET with a transabdominal sonography guide is superior to blind catheter placement [13]. With sonography we can ensure that the catheter passes the internal os and placement is $1-1.5 \mathrm{~cm}$ under the fundus.

Clinical experience demonstrates that UET is a safe technique but a report of mock ET showed that in 23\% of transfers, expulsion of catheter contents occurred or increased junctional zone contraction may decrease the pregnancy rate [14]. Transmission of vaginal microorganisms to the uterus via the transfer catheter may also decrease the pregnancy rate. All of these factors may be prevented by tubal transfer.

In the Van Voorhis et al. study comparing pregnancy rates after tubal and uterine transfer of cryopreserved embryos, tubal transfer improved the pregnancy rate. They reported clinical pregnancy rate of $68 \%$ and ongoing pregnancy rate of $58 \%$ per transfer with ZIFT compared with $24 \%$ and 19\% respectively with UET [15]. They

\begin{tabular}{|c|c|c|c|c|c|}
\hline \multirow[t]{2}{*}{ Variable } & \multicolumn{2}{|c|}{ ZIFT } & \multicolumn{2}{|c|}{ UET } & \multirow[t]{2}{*}{$P$} \\
\hline & No. & $\%$ & No. & $\%$ & \\
\hline Pregnancies that went to term & $25 / 36$ & 69 & $13 / 20$ & 65 & NS \\
\hline Multiple pregnancies & $20 / 43^{a}$ & 46 & $9 / 21^{b}$ & 42 & NS \\
\hline
\end{tabular}

${ }^{a}$ Twin 15 ; triplet 5.

${ }^{b}$ Twin 7 ; triplet 2

$N S=$ not significant $(P>0.05)$ 
suggested that tubal transfer of the cryopreserved embryo mimics a natural conception cycle in terms of early embryo development and implantation.

It has been reported that zygote intrafallopian transfer is a beneficial mode for treatment for patients with repeated failure of implantation in IVF and UET [16]. Pregnancy rates and implantation rates were significantly higher in the ZIFT group than in the UET group. They showed that patients in whom only low quality embryos are achieved in repeated IVF attempts can also benefit from the ZIFT procedure].

The limitations of this study include the small sample size and the fact that it was carried out in a single centre.
In summary, our results demonstrate a significant advantage of tubal over uterine transfer. Although the ZIFT approach involves greater cost and complexity, the greater pregnancy rate per cycle is one of the most important reasons for suggesting ZIFT to couples who have limited time and adequate financial support.

\section{References}

1. Balmaceda JP et al. Successful in vitro fertilization and embryo transfer in cynomolgus monkeys. Fertility and sterility, 1984, 42:791-5.

2. Devroey P et al. Pregnancy after translaparoscopic zygote intrafallopian transfer in a patient with sperm antibodies. Lancet, 1986, 1:1329.

3. Asch R. Uterine versus tubal embryo transfer in the human. Annals of the New York Academy of Science, 1991, 626:461-6.

4. Yovich JL et al. The relative chance of pregnancy following tubal or uterine transfer procedure. Fertility and sterility, 1988, 49:858-64.

5. Hammitt DG et al Comparison of concurrent pregnancy rates for in vitro fertilization-embryo transfer, pronuclear stage embryo transfer and gamete intra-fallopian transfer. Human reproduction, 1990, 5:947-54.

6. Pool TB et al. Zygote intrafallopian transfer as a treatment for nontubal infertility a 2-year study. Fertility and sterility, 1990, 54:482-8.

7. Tanbo T, Dale PO, Abyholm T. Assisted fertilization in infertile women with patent fallopian tubes. A comparison of in-vitro fertilization, gamete intra-fallopian transfer and tubal embryo stage transfer. Human reproduction, 1990, 5:266-70.

8. Fluker MR, Zouves CG, Bebbington MW. A prospective randomized comparison of zygote intrafallopian transfer and in vitro fertilization embryo transfer and in vitro fertilization-embryo transfer for nontubal factor infertility. Fertility and sterility, 1993, 60:515-9.
9. Preutthipan S et al. A prospective randomized crossover comparison of zygote intrafallopian transfer fallopian transfer and in vitro fertilization embryo transfer in unexplained infertility. Journal of the Medical Association of Thailand, 1994, 77:599-604.

10. Habana AE, Palter SF. Is tubal embryo transfer of any value, a meta analysis and comparison with the Society for Assisted Reproductive Technology database. Fertility and sterility, 2001, 76:286-92.

11. Levran D et al. Prospective evaluation of blastocyst stage transfer vs. zygote intrafallopian tube transfer in patients with repeated implantation failure. Fertility and sterility, 2002, 77:971-6.

12. Schulman JD. Delayed expulsion of transfer fluid after IVF/ET. Lancet, 1986, 1:44.

13. Woolcott R, Stanger J. Potentially important variables identified by transvaginal ultrasound-guided embryo transfer. $\mathrm{Hu}$ man reproduction, 1997, 12:963-6.

14. Mansour RT et al. Dummy embryo transfer using methylene blue dye. Human reproduction, 1994, 9:1257-9.

15. Van Voorhis BJ et al. Tubal versus uterine transfer of cryopreserved embryo. Fertility and sterility, 1995, 63:578-82.

16. Levran D et al. Zygote intrafallopian transfer may improve pregnancy rate in patients with repeated failure of implantation. Fertility and sterility, 1998, 69:26-30. 